\title{
Radiation pneumonitis in patients with non-small-cell lung cancer receiving chemoradiotherapy and an immune checkpoint inhibitor: a retrospective study
}

\author{
Jeong Yun Jang ${ }^{1}$, Su Ssan Kim ${ }^{1 *}$, Si Yeol Song ${ }^{1}$, Yeon Joo Kim', Sung-woo Kim² and Eun Kyung Choi ${ }^{1}$
}

\begin{abstract}
Background: Immunotherapy has been administered to many patients with non-small-cell lung cancer (NSCLC). However, only few studies have examined toxicity in patients receiving an immune checkpoint inhibitor (ICI) after concurrent chemoradiotherapy (CCRT). Therefore, we performed a retrospective study to determine factors that predict radiation pneumonitis (RP) in these patients.
\end{abstract}

Methods: We evaluated the size of the planning target volume, mean lung dose (MLD), and the lung volume receiving more than a threshold radiation dose (VD) in 106 patients. The primary endpoint was RP $\geq$ grade 2, and toxicity was evaluated.

Results: After CCRT, 51/106 patients were treated with ICl. The median follow-up period was 11.5 months (range, 3.0-28.2), and RP $\geq$ grade 2 occurred in 47 (44.3\%) patients: 27 and 20 in the ICl and non-ICl groups, respectively. Among the clinical factors, only the use of ICI was associated with RP $(p=0.043)$. Four dosimetric variables (MLD, V20, V30, and $\mathrm{V} 40$ ) had prognostic significance in univariate analysis for occurrence of pneumonitis (hazard ratio, $p$-value; MLD: 2.3, 0.009; V20: 2.9, 0.007; V30: 2.3, 0.004; V40: 2.5, 0.001). Only V20 was a significant risk factor in the non-ICI group, and MLD, V30, and V40 were significant risk factors in the ICI group. The survival and local control rates were superior in the ICl group than in the non-ICl group, but no significance was observed.

Conclusions: Patients receiving ICl after definitive CCRT were more likely to develop RP, which may be related to the lung volume receiving high-dose radiation. Therefore, several factors should be carefully considered for patients with NSCLC.

Keywords: Non-small-cell lung cancer, Concurrent chemoradiotherapy, Radiation therapy, Immunotherapy, Dosimetric factor, Tumor microenvironment

\footnotetext{
*Correspondence: watermountain@hanmail.net

${ }^{1}$ Department of Radiation Oncology, Asan Medical Center, University

of Ulsan College of Medicine, 88 Olympic-ro 43-gil, Songpa-gu, Seoul 05505, Republic of Korea

Full list of author information is available at the end of the article
}

\section{Background}

Non-small-cell lung cancer (NSCLC) is one of the leading causes of cancer mortality worldwide, and approximately $25-30 \%$ of patients are initially diagnosed with stage III, which is inoperable $[1,2]$. Definitive concurrent chemoradiotherapy (CCRT) was the standard treatment for locally advanced NSCLC, but it showed a short progression-free survival (PFS) of 8-11 months and overall original author(s) and the source, provide a link to the Creative Commons licence, and indicate if changes were made. The images or other third party material in this article are included in the article's Creative Commons licence, unless indicated otherwise in a credit line to the material. If material is not included in the article's Creative Commons licence and your intended use is not permitted by statutory regulation or exceeds the permitted use, you will need to obtain permission directly from the copyright holder. To view a copy of this licence, visit http://creativecommons.org/licenses/by/4.0/. The Creative Commons Public Domain Dedication waiver (http://creativeco mmons.org/publicdomain/zero/1.0/) applies to the data made available in this article, unless otherwise stated in a credit line to the data. 
survival (OS) of 20-23 months [3-5]. Many immunotherapy agents have been developed and have demonstrated promising results in locally advanced NSCLC patients, including immune checkpoint inhibitors (ICIs), which activate anti-tumor immune responses [6-8]. Notably, in the PACIFIC trial, the use of consolidative durvalumab after CCRT led to remarkable tumor control results. With the use of durvalumab, the median PFS was extended to 16.8 months versus 5.6 months with a placebo, and the 3-year OS rate with durvalumab and a placebo was $57.0 \%$ and $43.5 \%$, respectively $[9,10]$. Therefore, the use of adjuvant durvalumab in patients with unresectable NSCLC, whose conditions did not worsen after definitive CCRT, is now the new standard of care.

With the advent of the immunotherapy era, issues regarding the safety of treatment when administered with CCRT have emerged, although many studies have shown that the combination of ICI and CCRT is generally safe. However, in the PACIFIC trial, despite no observed significant differences, the incidence of any grade of pneumonitis was found to be $9.1 \%$ higher in the durvalumab group [9]. Radiation pneumonitis (RP) is a major complication in patients who have undergone thoracic irradiation, which can lead to a decreased quality of life, decreased performance status, and the early termination of further treatments; therefore, this needs to be carefully considered. Several studies have proven that some clinical and dosimetric factors are closely related to the occurrence of RP [11-13]. However, most have been conducted on CCRT-only groups; a few studies have targeted the patients who were additionally prescribed ICI after CCRT but only as a small cohort or as a single-arm study [14].

Therefore, we investigated the incidence of RP when ICI is used for any reason after definitive CCRT in patients with NSCLC who are unable to undergo surgery. In addition, we also sought to investigate which clinical and dosimetric factors are associated with the occurrence of RP.

\section{Methods}

\section{Patients}

We retrospectively reviewed the medical records of 143 consecutive NSCLC patients who underwent definitive CCRT with curative intent at Asan Medical Center from May 2018 to May 2020. Patients treated with ICI after CCRT were classified into the ICI group, and those who had never received ICI were classified into the non-ICI group. Among these patients, 37 were excluded from the analysis for the following reasons: patients with double primary cancer in the 5 years prior to the diagnosis of NSCLC $(n=7)$, distant metastasis at the time of diagnosis $(n=7)$, a history of previous lung surgery $(\mathrm{n}=1)$, a history of previous thoracic radiation therapy (RT) $(n=2)$, radiation dose $<45$ Gy $(n=1)$, use of $\mathrm{ICI}<2$ cycles $(\mathrm{n}=8)$, and short follow-up period after CCRT of $<3$ months $(n=11)$. Finally, 106 patients were enrolled in this study, and all of them underwent tissue biopsy, chest computed tomography (CT), 18-fluoro-deoxyglucose positron emission tomography (FDG-PET-CT), brain magnetic resonance imaging, and/or a pulmonary function test (PFT) before definitive treatment. The clinical stage of the patients was evaluated based on the 8th edition of the American Joint Committee on Cancer staging system.

\section{Treatments}

Radiation targeted the primary tumor and clinicallyinvolved lymph nodes. A lymph node was included in the gross tumor volume if the longest diameter was $>1 \mathrm{~cm}$ on its short axis by CT image and/or if the tumor had a hypermetabolic uptake on FDG-PET-CT or was proven to be a metastatic carcinoma by biopsy. No elective nodal irradiation was performed. A median total dose of $66 \mathrm{~Gy}$ (range, 46.0-73.0 Gy) was administered, and the fraction size was either 200 or 220 cGy. All patients received RT with the intensity-modulated radiotherapy technique. Radiation was delivered once daily (five times per week) with four to six coplanar or non-coplanar beams, and treatment verification was performed by weekly $\mathrm{kV}$ imaging guidance using set-up correction based on the carina and bony anatomy. The organs at risk included both lungs, the esophagus, the spinal cord, and the heart. The normal organ constraints included a maximal dose for the spinal cord of $<50 \mathrm{~Gy}$, a mean lung dose (MLD) of $<20 \mathrm{~Gy}$, a volume of lung receiving at least $20 \mathrm{~Gy}$ (V20) of $<30 \%$, a mean esophagus dose of $<35 \mathrm{~Gy}$, and a volume of heart receiving at least $60 \mathrm{~Gy}(\mathrm{~V} 60)<1 / 3, \mathrm{~V} 45<2 / 3$ and $\mathrm{V} 40<100 \%$. The following dosimetric parameters were generated from the dose-volume histogram (DVH) for the total lung: MLD, size of planning target volume (PTV), and the percentage of lung volume receiving more than a threshold radiation dose (VD), which refers to the relative lung volume receiving radiation above the threshold dose. As for the dose considered in VD, five variables were observed from 5 to 40 Gy (V5, V10, V20, V30, and V40).

Regarding chemotherapy, patients were prescribed paclitaxel-based paclitaxel and cisplatin (TP), paclitaxel and carboplatin (TC) regimen, an etoposide-based etoposide and cisplatin (EP), etoposide and carboplatin regimen, or cisplatin with pemetrexed. Of 106 patients, 51 received ICI after CCRT (Additional File 1). As the analysis was performed regardless of the treatment aim, all patients with consolidative, salvage, and palliative aims were enrolled together. PD-1/PDL-1 inhibitors were 
used as ICIs, including durvalumab, atezolizumab, pembrolizumab, and nivolumab. Durvalumab was prescribed at $10 \mathrm{mg} / \mathrm{kg}$ every 2 weeks, atezolizumab at $1200 \mathrm{mg}$ every 3 weeks, pembrolizumab at $200 \mathrm{mg}$ every 3 weeks, and nivolumab at $3 \mathrm{mg} / \mathrm{kg}$ every 2 weeks (all injected intravenously). All ICIs were discontinued when patients were determined to be intolerant due to adverse events or progressive disease.

\section{Follow-up and outcomes}

During the CCRT period, chest X-rays (CXR) and complete blood counts were performed weekly. All patients were regularly followed up by a radiation oncologist and a medical oncologist after the termination of CCRT. At each follow-up, history taking, physical examination, CXR, and blood tests were performed. Chest CT was performed every 6 months for 2 years and annually for 5 years.

The primary endpoint was $R P \geq$ grade 2 . $R P$ was assessed according to the toxicity criteria of the lung fibrosis established by Radiation Therapy Oncology Group and the European Organization for Research and Treatment of Cancer. The definitions were as follows: Grade 1, with no symptoms such as cough or shortness of breath and/or no opacity changes on chest CT; Grade 2 , with moderate symptoms which require outpatient steroid treatment and/or patchy image changes; Grade 3, with severe symptoms which need hospitalization and/ or increased density imaging changes; Grade 4, with severe symptoms requiring continuous $\mathrm{O}_{2}$ or assisted ventilation; Grade 5, expired. Based on the readings of the experienced chest radiologist, the RP was assessed by two radiation oncologists blindly on whether ICI was administered or not. Otherwise, all toxicity excluding RP, was assessed according to the National Cancer Institute's Common Terminology Criteria for Adverse Events ver. 5.0. The secondary endpoints were survival rate, locoregional failure (LRF) rate, distant metastasis (DM) rate, and acute and late toxicity. The LRF rate was evaluated by dividing cases into in-field and out-of-field failures based on whether the failure occurred inside or outside the PTV field. Recurrence in the ipsilateral lung was considered as LRF, and nodules in the contralateral lung or any pleural metastasis were considered as DM.

\section{Statistical analysis}

Statistical analyses were performed using SPSS version 22.0 (SPSS Inc., Chicago, IL, USA) and R version 3.6.1 (web-r.org). To compare the distribution of patient, disease, and treatment characteristics between the ICI group and non-ICI group, an independent-sample t-test was performed for continuous variables and chi-square test was performed for non-continuous variables. A receiver operating characteristic (ROC) curve was drawn to evaluate how well each DVH parameter could distinguish the incidence of RP, and through this, a suitable cut-off value and significance probability were calculated. Based on the cut-off values estimated from here, univariate and multivariate analyses for the occurrence of pneumonitis for the ICI and non-ICI groups were assessed with Fine and Gray competing risk regression analysis. P-values below 0.05 were considered statistically significant.

\section{Results}

\section{Patients' characteristics}

One hundred and six patients were included, and their characteristics are listed in Table 1 . The median age of the patients was 64 years (range, 38-82), and 84\% were male. Most patients showed a good Eastern Cooperative Oncology Group Performance Status of 0-1. The mean values of PFT did not differ significantly between the two groups. Approximately half of the patients with squamous cell carcinoma and adenocarcinoma were enrolled in the study. Most of the recruited patients (90.6\%) were stage III. The characteristics that showed differences between the two groups were age at the time of diagnosis, diffusing capacity of the lung for $\mathrm{CO}$, tumor location, and the type of chemotherapy agent used during CCRT, but none of these factors was a significant factor in the development of pneumonitis, as shown in subsequent analysis.

\section{Univariate and multivariate analyses for radiation pneumonitis}

The median follow-up period was 11.5 months (range, 3.0-28.2). During this period, $\mathrm{RP} \geq$ grade 2 occurred in 47 patients $(44.3 \%)$, and six $(5.7 \%)$ presented with $\mathrm{RP} \geq$ grade 3 . Univariate analysis was performed to determine the prognostic factors predicting $\mathrm{RP} \geq$ grade 2 . The results are shown in Table 2, and among the clinical factors, only the use of ICI was confirmed as a significant factor $(p=0.043)$. For the dosimetric factor, a cut-off value was set, and the hazard ratio (HR) was derived for the occurrence of pneumonitis, by dividing the group into patients with a value higher or lower than this. In univariate analysis for the entire cohort based on the cutoff value derived here, at an MLD of 16 Gy, V20, V30, and V40 showed a significant HR, which might increase the occurrence of RP (MLD, $p=0.009$; V20, $p=0.007$; V30, $p=0.004 ; \mathrm{V} 40, p=0.001)$. Figure 1 shows the cumulative incidence according to the use of ICI and the size of V40, which was the most significant factor among various dosimetric factors. In multivariate analysis, the use of ICI, MLD, and V40 were identified as significant factors. However, values from V5 to V40 and MLD were highly 


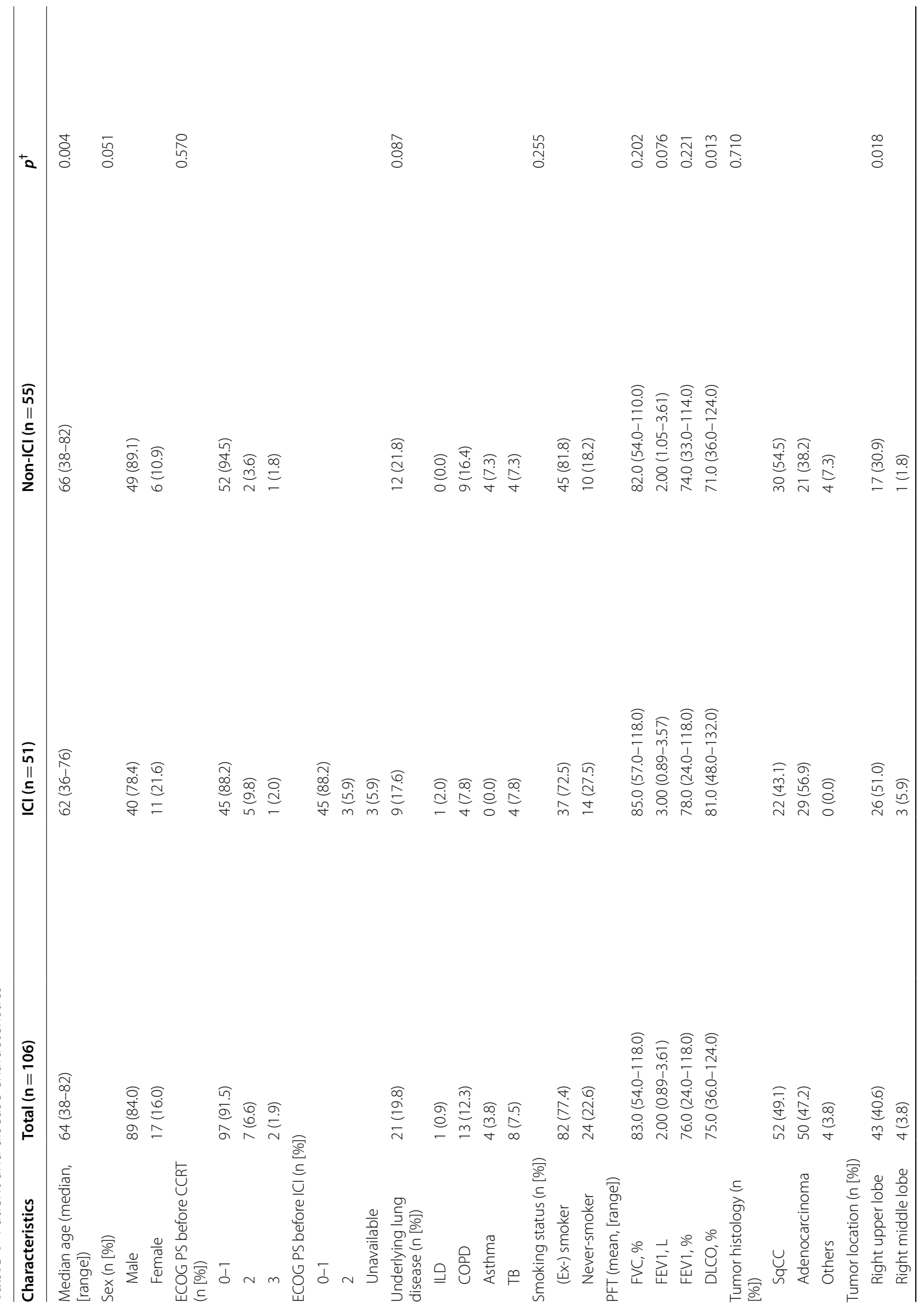




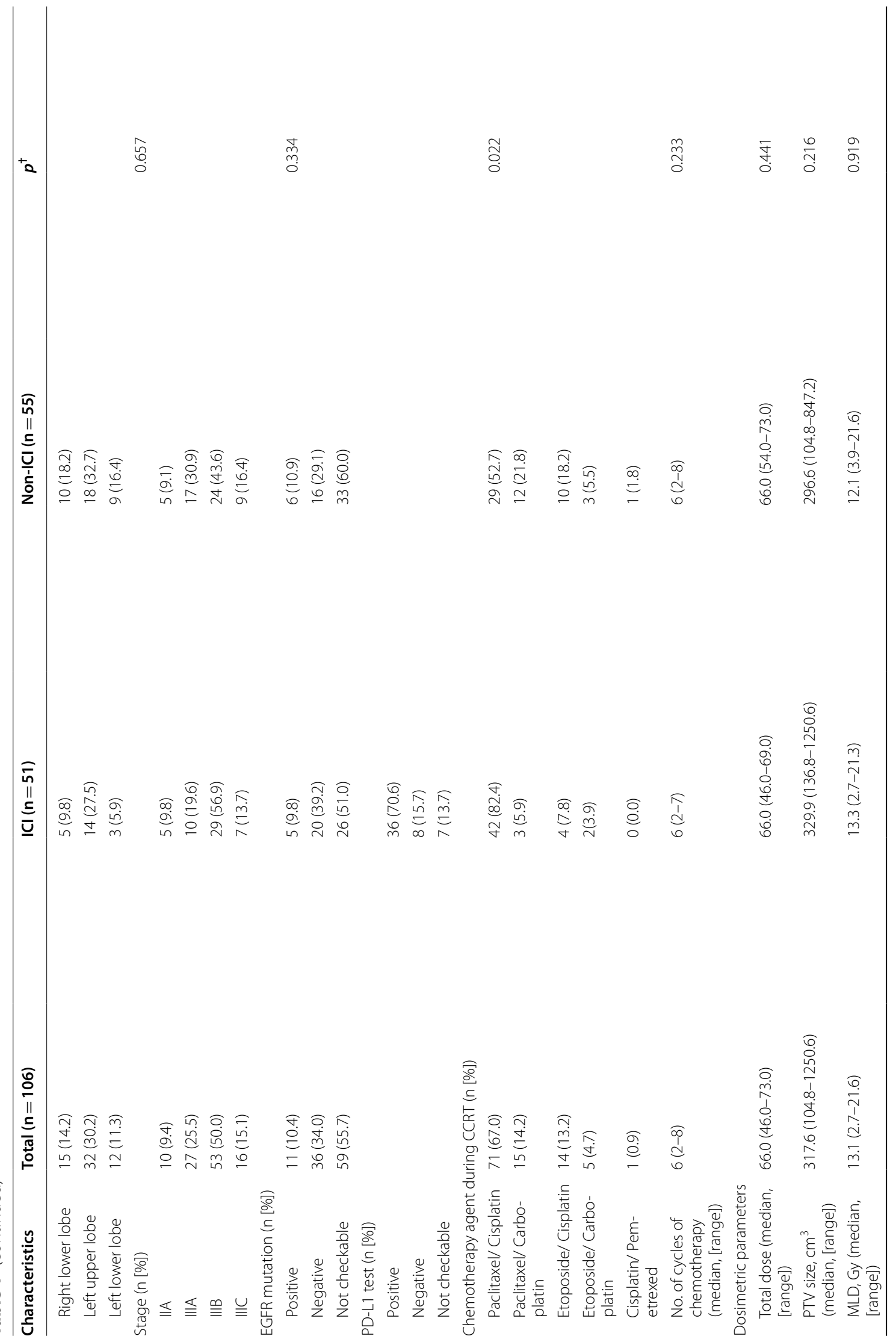




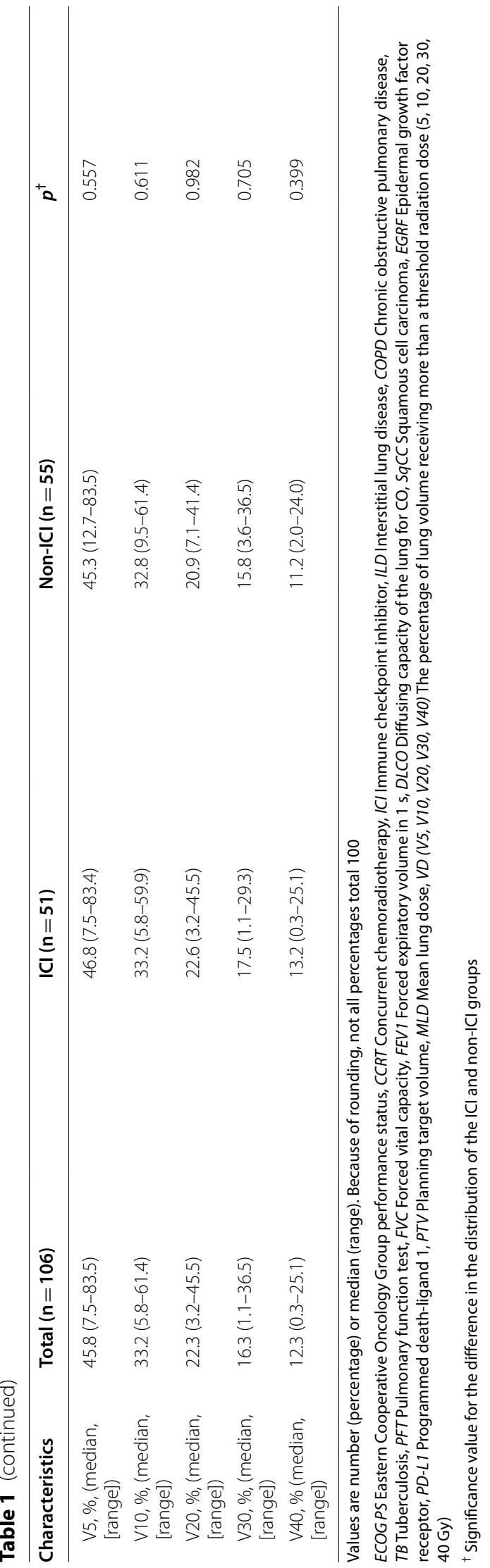


Table 2 Clinical and dosimetric factors predicting radiation pneumonitis $\geq$ grade 2 for patients treated with concurrent chemoradiotherapy

\begin{tabular}{|c|c|c|c|}
\hline Variable & HR $(95 \% \mathrm{Cl})$ & $p$ & Comparison group \\
\hline \multicolumn{4}{|l|}{ Clinical factors } \\
\hline Age, $\geq 65$ years & $1.136(0.644-2.004)$ & 0.660 & $<65$ \\
\hline ECOG PS before CCRT, $\geq 2$ & $0.421(0.102-1.744)$ & 0.233 & $<2$ \\
\hline ECOG PS before $|\mathrm{Cl}| \geq 2^{+}$ & $1.754(0.360-8.541)$ & 0.487 & \\
\hline (Ex-) smoker & $1.356(0.698-2.633)$ & 0.369 & Never-smoker \\
\hline Underlying lung disease & $1.162(0.587-2.300)$ & 0.668 & No underlying lung disease \\
\hline EGFR mutation & $1.155(0.445-2.998)$ & 0.767 & Wild type \\
\hline \multicolumn{4}{|l|}{ PFT } \\
\hline FVC, \% & $0.992(0.973-1.1012)$ & 0.428 & [Continuous] \\
\hline FEV1, L & $0.930(0.579-1.493)$ & 0.763 & [Continuous] \\
\hline FEV1, \% & $0.991(0.977-1.006)$ & 0.255 & [Continuous] \\
\hline DLCO, \% & 0.995 (0.980-1.010) & 0.487 & [Continuous] \\
\hline Clinical stage, $\geq I I I A$ & $1.358(0.503-3.664)$ & 0.546 & Stage II \\
\hline Tumor location, lower lobe & $2.014(0.951-4.266)$ & 0.067 & Tumor at RUL, RML, LUL \\
\hline Use of $\mathrm{ICl}$ & $1.802(1.019-3.186)$ & 0.043 & Non-ICl group \\
\hline $\begin{array}{l}\text { Chemotherapy agent during } \\
\text { CCRT, Paclitaxel/ Cisplatin }\end{array}$ & $1.498(0.834-2.690)$ & 0.177 & $\begin{array}{l}\text { Chemotherapy agent other } \\
\text { than Paclitaxel/ Cisplatin }\end{array}$ \\
\hline $\begin{array}{l}\text { No. of cycle of chemo- } \\
\text { therapy }\end{array}$ & $0.903(0.764-1.068)$ & 0.234 & [Continuous] \\
\hline \multicolumn{4}{|l|}{ Dosimetric factors } \\
\hline Total dose, > $60 \mathrm{~Gy}$ & $1.084(0.615-1.910)$ & 0.782 & $\leq 60 \mathrm{~Gy}$ \\
\hline PTV volume, $\geq 280 \mathrm{~cm}^{3}$ & $1.459(0.815-2.612)$ & 0.204 & $<280 \mathrm{~cm}^{3}$ \\
\hline$M L D, \geq 16 \mathrm{~Gy}$ & $2.299(1.234-4.284)$ & 0.009 & $<16 \mathrm{~Gy}$ \\
\hline$V 5, \geq 28 \%$ & $2.359(0.880-6.322)$ & 0.088 & $<28 \%$ \\
\hline$V 10, \geq 23 \%$ & $1.914(0.851-4.304)$ & 0.116 & $<23 \%$ \\
\hline$V 20, \geq 19 \%$ & $2.898(1.331-6.309)$ & 0.007 & $<19 \%$ \\
\hline V30, $\geq 18 \%$ & $2.295(1.297-4.060)$ & 0.004 & $<18 \%$ \\
\hline$V 40, \geq 14 \%$ & $2.513(1.431-4.412)$ & 0.001 & $<14 \%$ \\
\hline
\end{tabular}

HR Hazard ratio, Cl confidence interval, ECOG PS Eastern Cooperative Oncology Group performance status, CCRT Concurrent chemoradiotherapy, ICI Immune checkpoint inhibitor, EGRF Epidermal growth factor receptor, PFT Pulmonary function test, FVC Forced vital capacity, FEV1 Forced expiratory volume in $1 \mathrm{~s}$, DLCO Diffusing capacity of the Lung for CO, RUL Right upper lobe, RML Right middle lobe, LUL Left upper lobe, PTV Planning target volume, MLD Mean lung dose, VD (V5, $V 10$, V20, V30, V40) The percentage of lung volume receiving more than a threshold radiation dose (5, 10, 20, 30, 40 Gy)

${ }^{\dagger}$ Only 51 patients from the $\mathrm{ICl}$ group

correlated with each other, so they were not included in one analysis model as covariates and examined separately. Also, only V40, which had the highest significance, was selected for analysis on behalf of several VD values (Additional File 2).

\section{Radiation pneumonitis in $\mathrm{ICl}$ and non- $\mathrm{ICl}$ groups}

The incidence of $\mathrm{RP} \geq$ grade 2 was $52.9 \%$ for the ICI group, which was approximately $16 \%$ higher than that of the non-ICI group $(p=0.043)$ (Table 3). Severe $\mathrm{RP} \geq$ grade 3 occurred in three patients in both groups, and the incidence in the ICI and non-ICI groups was $5.9 \%$ and $5.4 \%$, respectively $(p=0.924)$. The interval between the end of CCRT and the occurrence of complications was 2.3 months for the ICI group and 3.7 months for the non-ICI group, with no significant difference $(p=0.782)$. In addition, the duration of steroid use due to pneumonitis was 56 days in the ICI group and 67 days in the non-ICI group, which was also similar in both groups with no significant difference $(p=0.848)$.

To establish the dosimetric factors that can predict the $\mathrm{RP}$ in each group, a univariate analysis was performed with the same cut-off value used for the entire cohort, and the results are shown in Table 4. In the ICI group, at an MLD of $16 \mathrm{~Gy}, \mathrm{~V} 30$ and V40 could significantly predict the occurrence of RP, and in the non-ICI group, only V20 could significantly predict RP. Therefore, we plotted the cumulative incidence of RP with V40, which showed the most significant relationship in the ROC curve and univariate analysis. It was found that the higher the value 

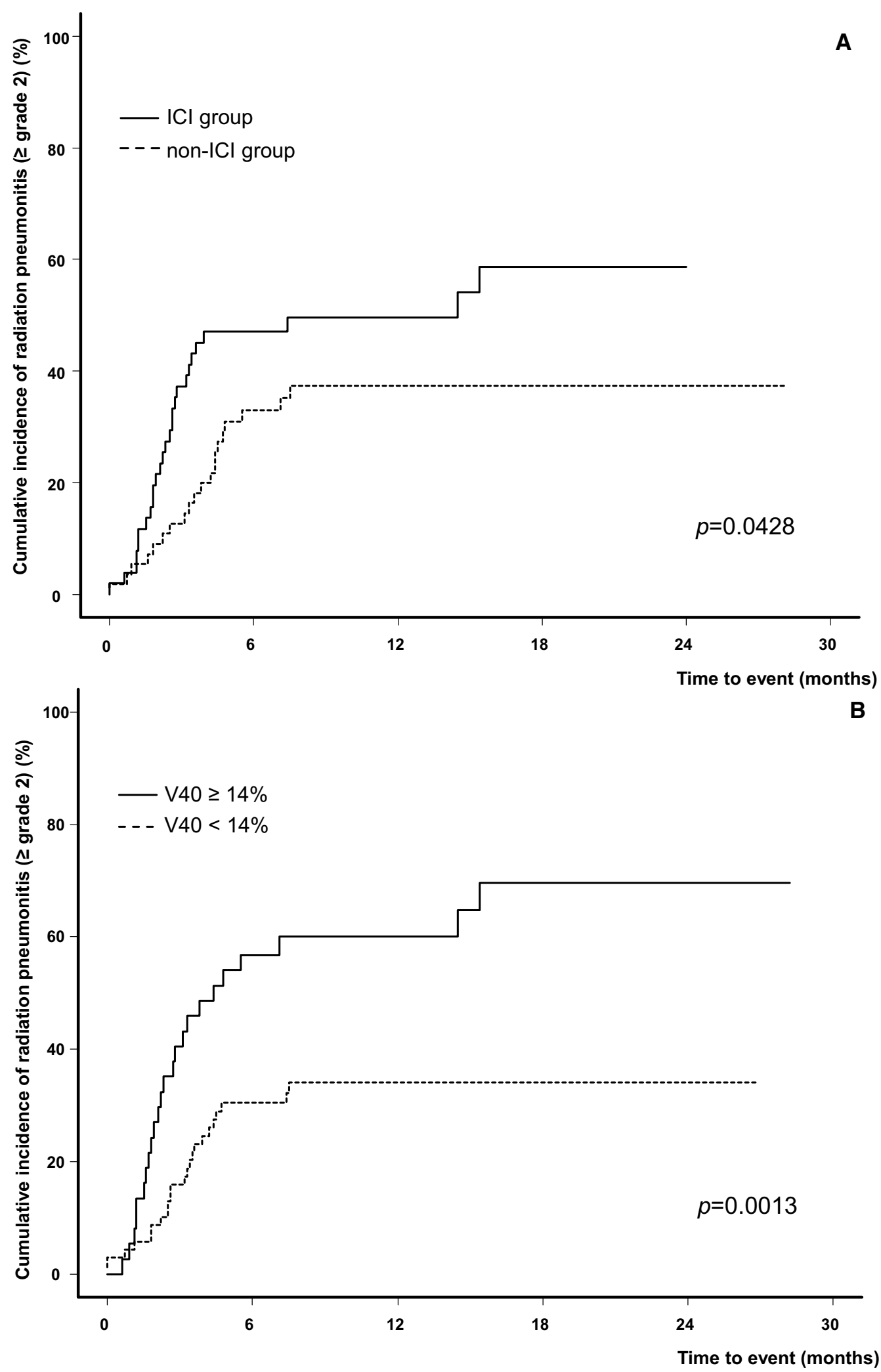

Fig. 1 Cumulative incidence of radiation pneumonitis $\geq$ grade 2 according to time to event between $\mathbf{a} \mid \mathrm{Cl}$ and non-ICl groups and $\mathbf{b} \mathrm{V} 40 \geq 14 \%$ and $<14 \%$. ICI Immune checkpoint inhibitor. V40The percentage of lung volume receiving more than $40 \mathrm{~Gy}$ 
Table 3 Characteristics of radiation pneumonitis according to immune checkpoint inhibitor administration

\begin{tabular}{|c|c|c|c|}
\hline Characteristics & $\mathrm{ICI}(\mathrm{n}=51)$ & Non-ICI $(n=55)$ & $p$ \\
\hline Patients with $\mathrm{RP} \geq$ grade 2 (n [\%]) & $27(52.9)$ & $20(36.4)$ & 0.043 \\
\hline Patients with $\mathrm{RP} \geq$ grade 3 (n [\%]) & $3(5.9)$ & $3(5.4)$ & 0.924 \\
\hline Onset time from end of CCRT, month (median, [range] $)^{\dagger}$ & $2.3(0.0-15.4)$ & $3.7(0.0-7.5)$ & 0.782 \\
\hline Onset time from the start of $\mathrm{ICl}$, month (median, [range] $)^{\dagger}$ & $1.4(0.0-5.8)$ & - & - \\
\hline Duration of steroid use, day (median, [range] $)^{+}$ & $56(16-238)$ & $67(6-114)$ & 0.848 \\
\hline
\end{tabular}

$R P$ Radiation pneumonitis, ICI Immune checkpoint inhibitor, CCRT Concurrent chemoradiotherapy

${ }^{\dagger}$ Only for patients with RT pneumonitis $\geq$ grade 2

of $\mathrm{V} 40$, the greater the difference in the cumulative incidence between the two groups (Fig. 2). The cumulative incidence of patients with a V40 value of $0-20 \%$ was $45.1 \%$ in the ICI group and $34.5 \%$ in the non-ICI group, showing a difference of approximately $10.6 \%$.

We also examined whether the interval between the end of CCRT and the start of immunotherapy in the ICI group affected the occurrence of RP. As a result of comparing the incidence of pneumonitis with an interval of 3 months, $51.4 \%$ of those who started ICI within 3 months of CCRT and $57.1 \%$ of those who started after 3 months had $\mathrm{RP} \geq$ grade 2 , showing no significant difference $(p=0.712)$.

\section{Survival and toxicities other than radiation pneumonitis}

The survival and patterns of recurrence are shown in Additional File 3 . In this analysis, only 90 patients were included; we excluded 16 patients who received immunotherapy with palliative and salvage aim within the ICI group. Of the 90 patients, 15 died, with a crude survival rate of $83.3 \%$. LRF occurred in 27 patients $(30.0 \%)$, and DM occurred in 17 patients (18.9\%): 10 in the ICI group and 7 in the non-ICI group. The most frequent metastasis sites were the bone, the liver, and the brain, in order of frequency.

Toxicity data for all patients are shown in Additional File 4 . For acute toxicity, 58 events occurred in 40 patients within the ICI group, and 39 events occurred in 23 patients within the non-ICI group. RP occurred most frequently, followed by esophagitis and dermatitis. In terms of late toxicity, 19 events occurred in 19 patients in the ICI group, and 21 events occurred in 21 patients in the non-ICI group. RP occurred most frequently, and peripheral neuropathy and pneumothorax were rare complications. Ten out of 106 patients $(9.4 \%)$ had acute toxicity $\geq 3$ grade, and three patients $(2.8 \%)$ had late toxicity $\geq$ grade 3 . Most patients with acute toxicity $\geq$ grade 3 recovered after various treatments such as antibiotics, steroid treatment, or esophageal stent insertion, but one patient who suffered brachial plexopathy did not improve despite the rehabilitation program. All three patients with late toxicity $\geq$ grade 3 experienced RP and seemed to improve with inpatient treatment, but two of them died from combined pneumonia and poor lung function.

\section{Discussion}

Several small cohort studies have reported the occurrence of RP in patients with NSCLC after the use of ICI with CCRT. In a study by Narek et al., RP $\geq$ grade 2 occurred in $18 \%$ of patients using durvalumab after CCRT, which was significantly higher than that of the CCRT-only group, which was only $9 \%(p=0.09)$ [15]. A single-arm study by Moore et al. found that $54 \%$ of patients who took durvalumab after CCRT had RP $\geq$ grade 2 [14]. In our study, the incidence was $16.5 \%$ higher in the ICI group than the non-ICI group, showing a similar tendency to the previous studies (ICI group $52.9 \%$ vs. non-ICI group $36.4 \%$, $p=0.043)$. In the PACIFIC trial, any grade of RP was $9.1 \%$ higher in the ICI group (durvalumab group $33.9 \%$ vs. placebo group $24.8 \%$ ), and the difference in the incidence of $\mathrm{RP}$ between the treatment groups was slightly greater in our study. However, for $\mathrm{RP} \geq$ grade 3, similar incidence rates were observed between the two groups in both our study and the PACIFIC study [16].

In general, $\mathrm{RP} \geq$ grade 2 is known to occur in $10-40 \%$ of patients with NSCLC who undergo CCRT with conventional fraction [17]. In our study, $44.3 \%$ of patients developed $R P \geq$ grade 2 , a higher incidence than that observed in other studies. There are likely to be several reasons for this result. First, the PACIFIC trial subgroup analysis found that Asians and patients with EGFR mutations had a high incidence of RP [16]. As the participants in our study were all Koreans, racial factors could explain the high RP incidence. Second, a high percentage of patients had EGFR mutations in this study; even if the mutation itself was not identified as a risk factor in univariate analysis in this study, in the PACIFIC trial, mutations were found in $8.2 \%$ of those tested and in $23.4 \%$ in this study. Third, patients who received neoadjuvant chemotherapy before CCRT were not included in this study. If the tumor size is reduced through induction chemotherapy, it can be expected that the radiation administered to the normal lung can also be reduced. In 







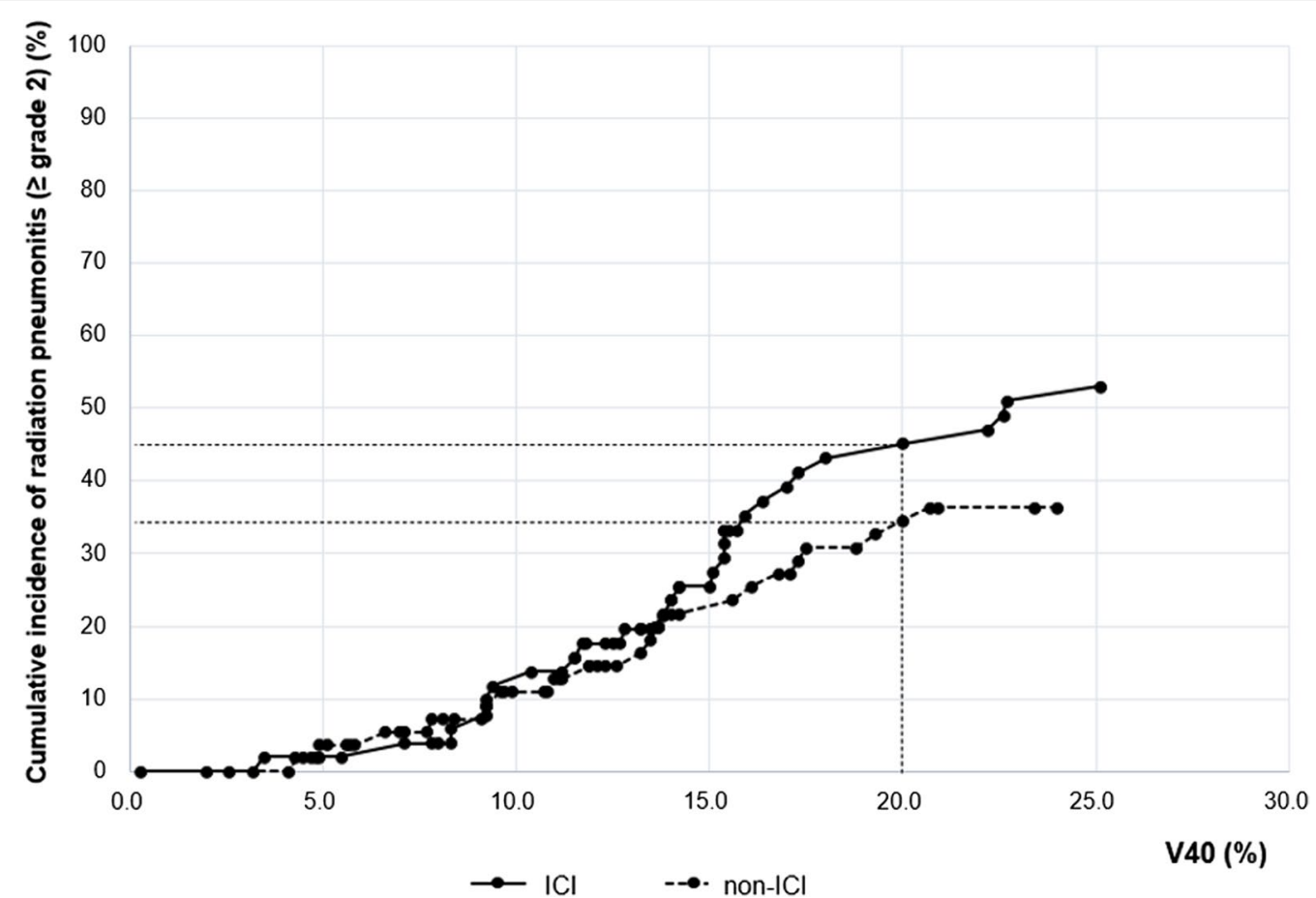

Fig. 2 Cumulative incidence of radiation pneumonitis $\geq$ grade 2 by $\mathrm{V} 40$. ICI Immune checkpoint inhibitor

the PACIFIC trial, approximately a quarter of patients underwent induction treatment before CCRT. Finally, the high incidence of RP in the current study may be due to the early initiation of steroid therapy when the patient complained of a mild cough or dyspnea, which might be less associated with RP.

Some studies have indicated that clinical factors, including the presence of chronic lung disease, stage IIIA or higher disease, tumors located in the lower lobe, and previous history of RT, can significantly increase the probability of $\mathrm{RP} \geq$ grade 2 [13]. However, none of these were found to be associated with the occurrence of RP in our study. Among clinical factors, only the use of ICI was found to be a significant factor. On the other hand, we found that initiating ICI at any point after the end of CCRT did not affect the development of RP; thus, it would be unnecessary to deliberately initiate immunotherapy late due to concerns about the potential side effects.

In our study, only V20 was identified as a factor correlated with RP in the non-ICI group. However, in the ICI group, significant HR were shown with V30 and V40, and the incidence of RP was found to increase in the high VD area. Usually, the lung volume irradiated with a high dose tends to correlate with target size. However, when observing the correlation between the PTV size and RP, the HR was 1.459, and there was no significant difference between the two groups; thus, V30 and V40 may have had a relationship with RP distinct from the field size. In addition, a similar study by Satoshi et al. also found that V30 had the largest AUC among various parameters in patients receiving adjuvant durvalumab after CCRT [18]. Previous studies have shown that sufficient lymphocytic infiltration is achieved through RT, which turns "cold" tumors "hot" and creates a tumor microenvironment (TME) that can respond well to various ICIs [19]. However, there remains controversy regarding the optimal dose and fraction scheme that can induce a change in the TME, and no definite conclusions have been drawn. In a preclinical study based on NSCLC cell line, the most potent $\mathrm{T}$ cell infiltration was induced by high-dose radiation; according to another study by Marconi et al., the probability of the occurrence of the abscopal effect based on changes in the immunologic environment is $50 \%$ with a biologically effective dose of at least 60 Gy [20, 21]. Therefore, there is a high possibility that a "hot" tumor environment will be created in the high dose area, which may be beneficial in terms of tumor cell control but is also the reason for the increased incidence of RP.

The survival and local control rate were superior in the ICI group, showing similar trends as those reported in the PACIFIC trial. However, these results were not significant, and we predict that this is due to an insufficient follow-up period of $<1$ year. In addition, patients 
with varying responses after CCRT were recruited into one group despite receiving different types of ICI, which makes it difficult to compare oncological outcomes directly. Future studies should be conducted on a larger number of patients with more uniform conditions and treatment characteristics, after which it is expected that results similar to those of other prospective studies will be obtained.

The limitations of this study were the small number of patients and the short follow-up time. Additionally, owing to the study's retrospective nature, the patient characteristics were somewhat different. Nonetheless, it would be of great significance to establish the frequency of adverse events in the new treatment combination and which factors affect them in the actual clinical situation.

\section{Conclusions}

The use of ICI after definitive CCRT is generally safe. However, it is important to be aware that the incidence of $\mathrm{RP} \geq$ grade 2 is slightly higher after ICI, and as this is associated with the lung volume irradiated with high doses of radiation, this should be fully considered in the treatment planning process for NSCLC patients.

\section{Abbreviations}

NSCLC: Non-small-cell lung cancer; CCRT: Concurrent chemoradiotherapy; PFS: Progression-free survival; OS: Overall survival; ICls: Immune checkpoint inhibitors; RP: Radiation pneumonitis; RT: Radiation therapy; CT: Computed tomography; FDG-PET-CT: 18-Fluoro-deoxyglucose positron emission tomography; PFT: Pulmonary function test; MLD: Mean lung dose; VD: Percentage of lung volume receiving more than a threshold dose; DVH: Dose-volume histogram; PTV: Planning target volume; CXR: Chest X-ray; LRF: Locoregional failure; DM: Distant metastasis; ROC: Receiver operating characteristic; HR: Hazard ratio; TME: Tumor microenvironment.

\section{Supplementary Information}

The online version contains supplementary material available at https://doi. org/10.1186/s13014-021-01930-2.

Additional file 1. Treatment characteristics of the $\mathrm{ICl}$ group.

Additional file 2. Multivariate analysis results using fine and gray competing risk regression analysis.

Additional file 3. Oncologic outcomes for 90 patients with non-small cell lung cancer treated with concurrent chemoradiotherapy.

Additional file 4. Toxicity results for 106 patients with non-small cell lung cancer treated with concurrent chemoradiotherapy.

\section{Acknowledgements}

None of the authors have any personal or financial conflicts of interest to declare.

\section{Authors' contributions}

JYJ, SSK, SYS, YJK, EKC: study concept and design. JYJ, SK: Acquisition of data. JYJ, SSK, YJK: Analysis and interpretation of data. JYJ, YJK Writing, review and/ or revision of manuscript. SK: administrative, technical, or material support. All authors read and approved the final manuscript.

\section{Funding}

This work was supported by a National Research Foundation (NRF) grant funded by the Korean government (NRF-2018R1D1A1B07049970).

\section{Availability of data and materials}

The data that support the findings of this study are available on request from the corresponding author.

\section{Declarations}

\section{Ethics approval and consent to participate}

This study was conducted in accordance with the 1964 Declaration of Helsinki. In addition, copies of written informed consent and Institutional Review Board (IRB) approval for clinical research have been kept. If necessary, the editor or reviewers may request copies of these documents to resolve questions about IRB approval and study conduct.

\section{Consent for publication}

Not applicable.

\section{Competing interests}

The authors declare that they have no competing interests.

\section{Author details}

${ }^{1}$ Department of Radiation Oncology, Asan Medical Center, University of Ulsan College of Medicine, 88 Olympic-ro 43-gil, Songpa-gu, Seoul 05505, Republic of Korea. ${ }^{2}$ Department of Radiation Oncology, Asan Medical Center, Seoul, Republic of Korea.

Received: 11 August 2021 Accepted: 12 October 2021

Published online: 04 December 2021

\section{References}

1. Bray F, Ferlay J, Soerjomataram I, Siegel RL, Torre LA, Jemal A. Global cancer statistics 2018: GLOBOCAN estimates of incidence and mortality worldwide for 36 cancers in 185 countries. CA Cancer J Clin. 2018;68(6):394-424.

2. Walters S, Maringe C, Coleman MP, Peake MD, Butler J, Young N, et al. Lung cancer survival and stage at diagnosis in Australia, Canada, Denmark, Norway, Sweden and the UK: a population-based study, 2004-2007. Thorax. 2013;68(6):551-64.

3. Hanna N, Neubauer M, Yiannoutsos C, McGarry R, Arseneau J, Ansari R, et al. Phase III study of cisplatin, etoposide, and concurrent chest radiation with or without consolidation docetaxel in patients with inoperable stage III non-small-cell lung cancer: the Hoosier Oncology Group and U.S. Oncology. J Clin Oncol. 2008;26(35):5755-60.

4. Segawa Y, Kiura K, Takigawa N, Kamei H, Harita S, Hiraki S, et al. Phase III trial comparing docetaxel and cisplatin combination chemotherapy with mitomycin, vindesine, and cisplatin combination chemotherapy with concurrent thoracic radiotherapy in locally advanced non-small-cell lung cancer: OLCSG 0007. J Clin Oncol. 2010:28(20):3299-306.

5. Yamamoto N, Nakagawa K, Nishimura Y, Tsujino K, Satouchi M, Kudo S, et al. Phase III study comparing second- and third-generation regimens with concurrent thoracic radiotherapy in patients with unresectable stage III non-small-cell lung cancer: West Japan Thoracic Oncology Group WJTOG0105. J Clin Oncol. 2010;28(23):3739-45.

6. Borghaei H, Paz-Ares L, Horn L, Spigel DR, Steins M, Ready NE, et al. Nivolumab versus docetaxel in advanced nonsquamous non-small-cell lung cancer. N Engl J Med. 2015;373(17):1627-39.

7. Herbst RS, Baas P, Kim DW, Felip E, Pérez-Gracia JL, Han JY, et al. Pembrolizumab versus docetaxel for previously treated, PD-L1-positive, advanced non-small-cell lung cancer (KEYNOTE-010): a randomised controlled trial. Lancet. 2016;387(10027):1540-50.

8. Rittmeyer A, Barlesi F, Waterkamp D, Park K, Ciardiello F, von Pawel J et al. Atezolizumab versus docetaxel in patients with previously treated non-small-cell lung cancer (OAK): a phase 3, open-label, multicentre randomised controlled trial. Lancet. 2017;389(10066):255-65. 
9. Antonia SJ, Villegas A, Daniel D, Vicente D, Murakami S, Hui R, et al. Durvalumab after chemoradiotherapy in stage III non-small-cell lung cancer. N Engl J Med. 2017;377(20):1919-29.

10. Gray JE, Villegas A, Daniel D, Vicente D, Murakami S, Hui R, et al. Three-year overall survival with durvalumab after chemoradiotherapy in stage III NSCLC-update from PACIFIC. J Thorac Oncol. 2020;15(2):288-93.

11. Boonyawan K, Gomez DR, Komaki R, Xu Y, Nantavithya C, Allen PK, et al. Clinical and dosimetric factors predicting grade $\geq 2$ radiation pneumonitis after postoperative radiotherapy for patients with non-small cell lung carcinoma. Int J Radiat Oncol Biol Phys. 2018;101(4):919-26.

12. Wang S, Liao Z, Wei X, Liu HH, Tucker SL, Hu CS, et al. Analysis of clinical and dosimetric factors associated with treatment-related pneumonitis (TRP) in patients with non-small-cell lung cancer (NSCLC) treated with concurrent chemotherapy and three-dimensional conformal radiotherapy (3D-CRT). Int J Radiat Oncol Biol Phys. 2006;66(5):1399-407.

13. Zhang XJ, Sun JG, Sun J, Ming H, Wang XX, Wu L, et al. Prediction of radiation pneumonitis in lung cancer patients: a systematic review. J Cancer Res Clin Oncol. 2012;138(12):2103-16.

14. Moore R, Lau S, Bezjak A, Sacher AG, Liu Z, Hope AJ, et al. The clinical relevance and management of grade 2 pneumonitis in Stage III non-small cell lung cancer patients on adjuvant durvalumab. International Journal of Radiation Oncology*Biology*Physics. 2020;108(3).

15. Shaverdian N, Thor M, Shepherd AF, Offin MD, Jackson A, Wu AJ, et al. Radiation pneumonitis in lung cancer patients treated with chemoradiation plus durvalumab. Cancer Med. 2020;9(13):4622-31.

16. Vansteenkiste J, Naidoo J, Faivre-Finn C, Özgüroğlu M, Villegas A, Daniel D, et al. MA05.02 PACIFIC subgroup analysis: pneumonitis in stage III, unresectable NSCLC patients treated with durvalumab vs. placebo after CRT. J Thoracic Oncol. 2018;13(10):S370-S1

17. Rodrigues $G$, Lock M, D'Souza D, Yu E, Van Dyk J. Prediction of radiation pneumonitis by dose - volume histogram parameters in lung cancer-a systematic review. Radiother Oncol. 2004;71 (2):127-38.

18. Saito S, Abe T, Kobayashi N, Aoshika T, Ryuno Y, Igari M, et al. Incidence and dose-volume relationship of radiation pneumonitis after concurrent chemoradiotherapy followed by durvalumab for locally advanced nonsmall cell lung cancer. Clin Transl Radiat Oncol. 2020;23:85-8.

19. Demaria S, Coleman CN, Formenti SC. Radiotherapy: changing the game in immunotherapy. Trends Cancer. 2016;2(6):286-94.

20. Marconi R, Strolin S, Bossi G, Strigari L. A meta-analysis of the abscopal effect in preclinical models: Is the biologically effective dose a relevant physical trigger? PLoS One. 2017;12(2):e0171559.

21. Zeng H, Zhang W, Gong Y, Xie C. Radiotherapy activates autophagy to increase CD8(+) T cell infiltration by modulating major histocompatibility complex class-l expression in non-small cell lung cancer. J Int Med Res. 2019;47(8):3818-30.

\section{Publisher's Note}

Springer Nature remains neutral with regard to jurisdictional claims in published maps and institutional affiliations.

Ready to submit your research? Choose BMC and benefit from:

- fast, convenient online submission

- thorough peer review by experienced researchers in your field

- rapid publication on acceptance

- support for research data, including large and complex data types

- gold Open Access which fosters wider collaboration and increased citations

- maximum visibility for your research: over $100 \mathrm{M}$ website views per year

At BMC, research is always in progress.

Learn more biomedcentral.com/submissions 